DESY 10-166

\title{
Inflation in string theory: a graceful exit to the real world
}

\author{
Michele Cicoli ${ }^{1}$, and Anupam Mazumdar 2, 3 \\ 1 Deutsches Elektronen-Synchrotron DESY, Notkestrasse 85, 22603 Hamburg, Germany \\ ${ }_{2}$ Physics Department, Lancaster University, Lancaster, LA1 4YB, United Kingdom \\ ${ }^{3}$ Niels Bohr Institute, Copenhagen University, Blegdamsvej-17, DK-2100, Denmark
}

\begin{abstract}
The most important criteria for a successful inflation are to explain the observed temperature anisotropy in the cosmic microwave background radiation, and exiting inflation in a vacuum where it can excite the Standard Model quarks and leptons required for the success of Big Bang Nucleosynthesis. In this paper we provide the first ever closed string model of inflation where the inflaton couplings to hidden sector, moduli sector, and visible sector fields can be computed, showing that inflation can lead to reheating the Standard Model degrees of freedom before the electro-weak scale.
\end{abstract}

\section{INTRODUCTION}

The inflationary paradigm has played a key rôle in explaining the large scale structure of the Universe and the temperature anisotropy in the cosmic microwave background $(\mathrm{CMB})$ radiation [1]. It is believed that a scalar field, the inflaton, drives the inflationary dynamics. During inflation the quantum fluctuations of the inflaton seed the initial perturbations for the structure formation, and after inflation its coherent oscillations lead to reheat the Universe with the observed light degrees of freedom of the Standard Model quarks and leptons (for a review see [2]). These degrees of freedom need also to thermalise before Big Bang Nucleosynthesis (BBN) 3].

In this regard visible sector models of inflation where inflation ends in the Standard Model gauge invariant vacuum, are preferred with respect to the ones where the inflaton belongs to the hidden sector and couples to all particles in the model. In the former case, there are very few models based on low scale supersymmetry (SUSY) as in the Minimal Supersymmetric Standard Model (MSSM), where there exist only 2 inflaton candidates which carry Standard Model charges [4], and their decay populates the Universe with MSSM particles and dark matter [5].

On the contrary there are many models of inflation where the inflaton is a Standard Model gauge singlet [2]. Promising examples are closed string inflationary models in type IIB flux compactifications [6, 7]. The inflaton is a Kähler modulus parameterising the size of an internal cycle, and inflation can be embedded within an UV complete theory where the $\eta$-problem can be solved due to the no-scale structure of the potential and the fact that the volume mode of the internal manifold is kept stable during inflation. Moreover the order of magnitude of all the inflaton couplings can be computed [8, 9].

Despite all these successes, it is still hard to explain how the inflaton energy gets transferred primarily to visible degrees of freedom, and not to hidden ones, since $a$ priori a gauge singlet inflaton has no preference to either the visible or the hidden sector.

Thus the main challenge to build any hidden sector model of inflation is to reheat the visible sector so that the thermal bath prior to BBN is filled with the Standard
Model hadrons.

In order to fulfill this, the conditions are:

- The inflaton must decay primarily into the visible sector, and its coupling to the hidden sector must be weak enough to prevent an overproduction of its degrees of freedom non-thermally or nonperturbatively as in the case of preheating (for a review see [10]).

- The hidden sector must not contain very light species since their presence at the time of BBN could modify the light-element abundance. The current LEP and BBN constraints on extra light species is very tight, i.e. $\leq 4[3]$.

- The visible sector must be sequestered from the hidden sector so that the late decay of the hidden degrees of freedom does not spoil the success of $\mathrm{BBN}$ or overpopulate the dark matter abundance.

We stress that hidden sectors arise naturally in string compactifications since they come along with many internal cycles which have to be stabilised. This is generically achieved wrapping stacks of $D p$-branes around $(p-3)$-cycles in order to generate perturbative and nonperturbative effects that lead to moduli fixing. The presence of such $D p$-branes is also in general needed to achieve tadpole cancellation which ensures the absence of anomalies in the 4D effective field theory. Given that each stack of $D$-branes supports a different field theory, the presence of hidden sectors turns out to be very generic in any such constructions.

In order to address all these issues related to the hidden sector, it is crucial to know the inflaton couplings to all hidden and visible degrees of freedom. In this paper we shall present a type IIB closed string inflation model where all the inflaton couplings can be derived within an UV complete theory [8]. The knowledge of these couplings allows us to study the reheating of the visible degrees of freedom which can be achieved with a temperature above the BBN temperature (for other studies on (p)reheating in string cosmology see [11, 12]).

The beauty of this top-down setup is also that the flatness of the inflaton potential can be checked, and all the 
main phenomenological scales can be generated with the following achievements:

- correct amount of CMB density perturbations;

- right scale for grand unification theories (GUT);

- TeV scale SUSY;

- no cosmological moduli problem (CMP).

\section{TYPE IIB LARGE VOLUME SCENARIOS}

String compactifications typically give rise to a large number of moduli which can be ideal candidates to drive inflation. It is crucial to lift these flat directions in order to determine the features of the low energy effective field theory (like masses and coupling constants) and to avoid the presence of unobserved long range fifth forces.

\section{A. Moduli stabilisation}

Moduli stabilisation is best understood in the context of type IIB where background fluxes fix the dilaton and the complex structure moduli at tree-level. On the other hand, the stabilisation of the Kähler moduli requires to consider perturbative and non-perturbative effects $13-$ 15]. Expressing the Kähler moduli as $T_{i}=\tau_{i}+i b_{i}, i=$ $1, \ldots, h_{1,1}$, with $\tau_{i}$ the volume of an internal 4-cycle $\Sigma_{i}$ and $b_{i}=\int_{\Sigma_{i}} C_{4}$, we shall focus on compactification manifolds whose volume reads (with $\alpha>0, \gamma_{i}>0 \forall i$ ):

$$
\hat{\mathcal{V}}=\alpha\left(\tau_{1}^{3 / 2}-\gamma_{2} \tau_{2}^{3 / 2}-\gamma_{3} \tau_{3}^{3 / 2}-\gamma_{4} \tau_{4}^{3 / 2}\right)
$$

Assuming that the tadpole-cancelation condition can be satisfied by an appropriate choice of background fluxes, we wrap a hidden sector $D 7$-stack that undergoes gaugino-condensation both around $\tau_{2}$ and $\tau_{3}$. This brane set-up induces a superpotential of the form (the dilaton and the complex structure moduli are flux-stabilised at tree level and so they can be integrated out):

$$
W=W_{0}+A_{2} e^{-a_{2} T_{2}}+A_{3} e^{-a_{3} T_{3}} .
$$

We focus on the case when the cycle $\tau_{4}$ supporting the visible sector shrinks down at the singularity. The visible sector is built via $D 3$-branes at the quiver locus and the gauge coupling is given by the dilaton $s$ (with $\langle s\rangle=g_{s}^{-1}$ ) while $\tau_{4}$ enters as a flux-dependent correction: $4 \pi g^{-2}=$ $s+h(F) \tau_{4}$. The Kähler potential with the leading order $\alpha^{\prime}$ correction can be expanded around $\tau_{4}=0$ [16]:

$$
K=-2 \ln \left(\mathcal{V}+\frac{\xi s^{3 / 2}}{2}\right)+\lambda \frac{\tau_{4}^{2}}{\mathcal{V}}-\ln (2 s),
$$

with $\mathcal{V}=\alpha\left(\tau_{1}^{3 / 2}-\gamma_{2} \tau_{2}^{3 / 2}-\gamma_{3} \tau_{3}^{3 / 2}\right)$. In the absence of Standard Model singlets which can get a non-vanishing
VEV, an anomalous $U(1)$ on the visible sector cycle generates a D-term potential with a Fayet-Iliopoulos term:

$$
V_{D}=\left(\frac{2 \pi}{s+h(F) \tau_{4}}\right) \xi_{F I}^{2} \text { with } \xi_{F I}=\frac{Q_{\tau_{4}} \tau_{4}}{\mathcal{V}}
$$

while the leading order F-term potential reads (after minimising the axion directions):

$$
\begin{aligned}
V_{F}= & \sum_{i=2}^{3} \frac{8 a_{i}^{2} A_{i}^{2}}{3 \alpha \gamma_{i}} \frac{\sqrt{\tau_{i}} e^{-2 a_{i} \tau_{i}}}{\mathcal{V}}-4 W_{0} \sum_{i=2}^{3} a_{i} A_{i} \frac{\tau_{i} e^{-a_{i} \tau_{i}}}{\mathcal{V}^{2}} \\
& +\frac{3 \xi W_{0}^{2}}{4 g_{s}^{3 / 2} \mathcal{V}^{3}}
\end{aligned}
$$

The D-term potential scales as $V_{D} \sim \mathcal{O}\left(\mathcal{V}^{-2}\right)$, while in the regime $\mathcal{V} \sim e^{a_{i} \tau_{i}}, i=2,3, V_{F} \sim \mathcal{O}\left(\mathcal{V}^{-3}\right)$. Hence at leading order $\xi_{F I}=0$ leads to $\tau_{4} \rightarrow 0$ fixing this cycle at the quiver locus [16]. On the other hand, $V_{F}$ completely stabilises $\tau_{2}, \tau_{3}$ and the volume $\mathcal{V} \simeq \alpha \tau_{1}^{3 / 2}$ at:

$$
a_{i}\left\langle\tau_{i}\right\rangle=\frac{1}{g_{s}}\left(\frac{\xi}{2 \alpha J}\right)^{2 / 3} \text { with } J=\sum_{i=2}^{3} \gamma_{i} / a_{i}^{3 / 2},
$$

and $\langle\mathcal{V}\rangle=\left(\frac{3 \alpha \gamma_{i}}{4 a_{i} A_{i}}\right) W_{0} \sqrt{\left\langle\tau_{i}\right\rangle} e^{a_{i}\left\langle\tau_{i}\right\rangle}, \forall i=2,3$.

Moduli stabilisation is performed without fine tuning the internal fluxes $\left(W_{0} \sim \mathcal{O}(1)\right)$ and the volume is fixed exponentially large in string units. As a consequence, one has a very reliable effective field theory, as well as a tool for the generation of phenomenologically desirable hierarchies.

\section{B. Particle physics phenomenology}

The particle phenomenology is governed by the background fluxes, which break SUSY by the $F$-terms of the Kähler moduli and the dilaton which then mediate this breaking to the visible sector. However the $F$ term of $\tau_{4}$ vanishes since it is proportional to $\xi_{F I}=0$ : $F^{4} \sim e^{K / 2} K^{4 \overline{4}} W_{0} \xi_{F I}=0$. Thus there is no local SUSYbreaking and the visible sector is sequestered, implying that the soft terms can be suppressed with respect to $m_{3 / 2}$ by an inverse power of the volume. The main scales in the model are:

- GUT-scale: $M_{G U T} \sim M_{P} / \mathcal{V}^{1 / 3}$,

- String-scale: $M_{s} \sim M_{P} / \mathcal{V}^{1 / 2}$,

- Kaluza-Klein scale: $M_{K K} \sim M_{P} / \mathcal{V}^{2 / 3}$,

- Gravitino mass: $m_{3 / 2} \sim M_{P} / \mathcal{V}$,

- Blow-up modes: $m_{\tau_{i}} \sim m_{3 / 2}, i=2,3$,

- Volume mode: $m_{\mathcal{V}} \sim M_{P} / \mathcal{V}^{3 / 2}$,

- Soft-terms: $M_{\text {soft }} \sim m_{3 / 2}^{2} / M_{P} \sim M_{P} / \mathcal{V}^{2}$.

Setting the volume $\mathcal{V} \simeq 10^{6-7}$ in string units, corresponding to $M_{s} \simeq 10^{15} \mathrm{GeV}$, one can realise GUT theories, TeV scale SUSY and avoid any CMP [16]. 


\section{Inflationary cosmology}

A very promising inflationary model can be embedded in this type IIB scenario with the inflaton which is the size of the blow-up $\tau_{2}$ [6]. Displacing $\tau_{2}$ far from its minimum, due to the exponential suppression, this field experiences a very flat direction which is suitable for inflation. The other blow-up $\tau_{3}$, which sits at its minimum while $\tau_{2}$ is slow rolling, has been added to keep the volume stable during inflation. In terms of the canonically normalised inflaton $\phi$, the potential looks like [6]:

$$
V \simeq V_{0}-\beta\left(\frac{\phi}{\mathcal{V}}\right)^{4 / 3} e^{-a \mathcal{V}^{2 / 3} \phi^{4 / 3}}
$$

This is a model of small field inflation, and so no detectable gravity waves are produced: $r \equiv T / S \ll 1$. The spectral index is in good agreement with the observations: $0.960<n_{s}<0.967$, and the requirement of generating enough density perturbations fixes $\mathcal{V} \simeq 10^{6-7}$ which is the same value preferred by particle physics.

Potential problems come from $g_{s}$ corrections to $K$ [17] which dominate the potential spoiling its flatness once $\tau_{2}$ is displaced far from its minimum. The only way-out is to fine-tune these $g_{s}$ corrections small [8].

In principle the non-perturbative potential for $\tau_{2}$ could also be generated by a $D 3$-brane instanton. In this way hidden sectors and $g_{s}$ corrections would be absent. However the requirement of having $\mathcal{V} \simeq 10^{6-7}$ prevents this set-up since both $\tau_{2}$ and $\tau_{3}$ would be fixed smaller than the string scale where the effective field theory cannot be trusted anymore [8]. Thus we realise that hidden sectors are always present in these models.

\section{Hidden sector configurations}

The hidden sectors on $\tau_{i}, i=2,3$, consist in a supersymmetric field theory that undergoes gaugino condensation. Broadly speaking we can entertain 3 scenarios for the possible particle content and mass spectrum:

- The hidden sector is a pure $N=1$ supersymmetric Yang-Mills (SYM) theory which due to strong dynamics confines in the IR at the scale $\Lambda$ :

$$
\Lambda_{i}=M_{s} e^{-\left(4 \pi g^{-2}\right) a_{i} / 3}, i=2,3 .
$$

Given that $4 \pi g^{-2}=\tau_{i}$, and at the minimum $e^{-a_{i} \tau_{i}} \sim \mathcal{V}^{-1}$, the order of magnitude of $\Lambda_{i}$ can be estimated as $\Lambda_{i} \simeq M_{P} \mathcal{V}^{-5 / 6}$. The theory develops a mass gap and all particles acquire a mass of the order $\Lambda_{i}$ and are heavier than the inflaton after inflation since $m_{\tau_{2}} \simeq M_{P} / \mathcal{V}<\Lambda_{i}$. Thus the inflaton decay to hidden degrees of freedom is kinematically forbidden!

- The hidden sector is a pure SYM theory plus a massless $U(1)$. The mass-spectrum below $\Lambda$ consists of massless hidden photons and photini with an $\mathcal{O}\left(M_{\text {soft }}\right)$ mass due to SUSY-breaking effects.
- The hidden sector is an $N=1 S U\left(N_{c}\right)$ theory with $N_{f}<\left(N_{c}-1\right)$ flavours. The condensates of gauge bosons and gauginos get a mass of the order $\Lambda$ while all the matter condensates get an $\mathcal{O}\left(M_{\text {soft }}\right)$ mass due to SUSY-breaking effects except pion-like mesons which remain massless in the presence of spontaneous chiral symmetry breaking. If chiral symmetry is explicitly broken by a low energy Higgs-like mechanism, all matter fields get a $\delta m \ll M_{\text {soft }}$ correction to their masses. For an additional massless $U(1)$, there are also massless hidden photons and photini with an $\mathcal{O}\left(M_{\text {soft }}\right)$ mass.

\section{REHEATING}

We shall now focus on the study of reheating of the MSSM degrees of freedom after the end of inflation. In order to check what fraction of the inflaton energy is transferred to hidden and visible degrees of freedom, we have to derive the moduli mass spectrum and their couplings to all particles in the model.

\section{A. Canonical normalisation and mass spectrum}

The first step is to canonically normalise the moduli around the minimum of their VEVs: $\tau_{i}=\left\langle\tau_{i}\right\rangle+\delta \tau_{i}$, $\forall i$, The fluctuations $\delta \tau_{i}$ are written in terms of the canonically normalised fields $\delta \phi_{i}$ as $\delta \tau_{i}=\frac{1}{\sqrt{2}} C_{i j} \delta \phi_{j}$, where $C_{i j}$ are the eigenvectors of the matrix $\left(M^{2}\right)_{i j} \equiv$ $\frac{1}{2}\left(K^{-1}\right)_{i k} V_{k j}$ whose eigenvalues $m_{i}^{2}$ are the moduli mass-squareds.

The form of $K$, eq. (3), and $\left\langle\tau_{4}\right\rangle=0$ imply that at leading order $\tau_{2}$ does not mix with $\tau_{4}$. However $\tau_{2}$ mixes with $s$ due to $\alpha^{\prime}$ corrections to $K[8]$ :

$$
\begin{aligned}
\delta \tau_{1} & \sim \mathcal{O}\left(\mathcal{V}^{2 / 3}\right) \delta \phi_{1}+\sum_{i} \mathcal{O}\left(\mathcal{V}^{1 / 6}\right) \delta \phi_{i}, i=2,3, s \\
\delta \tau_{i} & \sim \mathcal{O}\left(\mathcal{V}^{1 / 2}\right) \delta \phi_{i}+\mathcal{O}(1) \delta \phi_{1}+\sum_{j} \mathcal{O}\left(\mathcal{V}^{-1 / 2}\right) \delta \phi_{j}, i=2,3 \\
\delta \tau_{4} & \sim \mathcal{O}\left(\mathcal{V}^{1 / 2}\right) \delta \phi_{4} \\
\delta s & \sim \mathcal{O}(1) \delta \phi_{s}+\mathcal{O}\left(\mathcal{V}^{-1 / 2}\right) \delta \phi_{1}+\sum_{i} \mathcal{O}\left(\mathcal{V}^{-1}\right) \delta \phi_{i}, i=2,3
\end{aligned}
$$

with $j=2,3, s, j \neq i$. The masses turn out to be:

$$
m_{1}^{2} \simeq \frac{M_{P}^{2}}{\mathcal{V}^{3}}, m_{i}^{2} \simeq \frac{M_{P}^{2}}{\mathcal{V}^{2}}, \forall i=2,3, s, \text { and } m_{4}^{2} \simeq \frac{M_{P}^{2}}{\mathcal{V}}
$$

\section{B. Inflaton couplings}

The inflaton coupling to visible and hidden degrees of freedom can be derived from the moduli dependence of the kinetic and mass terms of open string modes. The 
moduli are expanded around their VEVs and then expressed in terms of the canonically normalised fields. This procedure led to the derivation of the moduli couplings to all particles in the model [8, 9], finding that the strongest moduli decay rates are to hidden gauge bosons on $\tau_{2}$ and $\tau_{3}$, and to visible gauge bosons at the $\tau_{4}$-singularity (see Tab. I).

\begin{tabular}{c||c|c|c|c|c} 
& $\delta \phi_{1}$ & $\delta \phi_{2}$ & $\delta \phi_{3}$ & $\delta \phi_{4}$ & $\delta \phi_{s}$ \\
\hline \hline$\left(F_{\mu \nu}^{(2)} F_{(2)}^{\mu \nu}\right)$ & $\frac{1}{M_{P}}$ & $\frac{\mathcal{V}^{1 / 2}}{M_{P}}$ & $\frac{1}{\mathcal{V}^{1 / 2} M_{P}}$ & - & $\frac{1}{\mathcal{V}^{1 / 2} M_{P}}$ \\
\hline$\left(F_{\mu \nu}^{(3)} F_{(3)}^{\mu \nu}\right)$ & $\frac{1}{M_{P}}$ & $\frac{1}{\mathcal{V}^{1 / 2} M_{P}}$ & $\frac{\mathcal{V}^{1 / 2}}{M_{P}}$ & - & $\frac{1}{\mathcal{V}^{1 / 2} M_{P}}$ \\
\hline$\left(F_{\mu \nu}^{(4)} F_{(4)}^{\mu \nu}\right)$ & $\frac{1}{\mathcal{V}^{1 / 2} M_{P}}$ & $\frac{1}{\mathcal{V} M_{P}}$ & $\frac{1}{\mathcal{V} M_{P}}$ & $\frac{\mathcal{V}^{1 / 2}}{M_{P}}$ & $\frac{1}{M_{P}}$
\end{tabular}

TABLE I: Moduli couplings to all gauge bosons in the model.

\section{Moduli dynamics after inflation and reheating}

At the end of inflation, due to the steepness of the potential, the inflaton $\tau_{2}$, which acts like a homogeneous condensate, stops oscillating coherently around its minimum just after 2-3 oscillations due to a very violent nonperturbative production of $\delta \tau_{2}$ quanta [12]. The production of other degrees of freedom at preheating is instead less efficient.

According to the second of equations (9), our Universe is mostly filled with $\delta \phi_{2}$ plus some $\delta \phi_{1}$ and fewer $\delta \phi_{3}$ and $\delta \phi_{s}$-particles. Thus the energy density is dominated by $\delta \phi_{2}$ whose perturbative decay leads to reheating. Denoting as $g$ the visible gauge bosons and as $X_{2}$ and $X_{3}$ the hidden ones, the coupling of $\delta \phi_{2}$ to $X_{2} X_{2}$ is stronger than the one to $X_{3} X_{3}$ which, in turn, is stronger than the one to $g g$. This is due to the geometric separation between $\tau_{2}$ and $\tau_{3}$, and the sequestering of the visible sector at the $\tau_{4}$-singularity.

Hence the first decays are $\delta \phi_{i} \rightarrow X_{i} X_{i}, i=2,3$ with decay rate $\Gamma \sim M_{P} / \mathcal{V}^{2}$. Thus the inflaton dumps all its energy to hidden, instead of visible, degrees of freedom without reheating the visible sector. We stress that there is no direct coupling between hidden and visible degrees of freedom since they correspond to two open string sectors localised in different regions of the Calabi-Yau, and so the reheating of the visible sector cannot occur via the decay of hidden to visible degrees of freedom. Hence the only way-out is to forbid the decay of $\delta \phi_{2}$ to any hidden particle. This forces us to consider on both $\tau_{2}$ and $\tau_{3}$ a pure $N=1 \mathrm{SYM}$ theory that develops a mass gap, so that the decay of $\delta \phi_{2}$ to $X_{i} X_{i}, i=2,3$ is kinematically forbidden.

Then the first decay is $\delta \phi_{s} \rightarrow g g$ with $\Gamma \sim M_{P} / \mathcal{V}^{3}$ but without leading to reheating since the energy density is dominated by $\delta \phi_{2}$. Reheating occurs only later on when $\delta \phi_{2}$ decays to visible gauge bosons with total decay rate $\Gamma_{\delta \phi_{2} \rightarrow g g}^{T O T} \simeq(\ln \mathcal{V})^{3} M_{P} \mathcal{V}^{-5}$ [8]. At the same time $\delta \phi_{3} \rightarrow g g$ without giving rise to reheating since $\delta \phi_{3}$ is not dominating the energy density.
The maximal reheating temperature for the visible sector in the approximation of sudden thermalisation can be worked out equating $\Gamma_{\delta \phi_{2} \rightarrow g g}^{T O T}$ to $H \simeq\left(T_{R H}^{\max }\right)^{2} / M_{P}[8]$ :

$$
T_{R H}^{\max } \simeq \sqrt{\Gamma_{\delta \phi_{2} \rightarrow g g}^{T O T} M_{P}} \simeq(\ln \mathcal{V})^{3 / 2} \frac{M_{P}}{\mathcal{V}^{5 / 2}}
$$

For $\mathcal{V} \simeq 10^{6-7}$, we obtain $T_{R H}^{\max } \simeq 10^{2-4} \mathrm{GeV}$ which is higher than $T_{B B N} \simeq 1 \mathrm{MeV}$, and so it does not create any problem if the matter-antimatter asymmetry could be realised in a non-thermal/thermal way. Later on $\delta \phi_{1}$ decays to visible degrees of freedom out of thermal equilibrium without suffering from the CMP since its decays before $\mathrm{BBN}: T_{\delta \phi_{1} \rightarrow g g} \simeq M_{P} \mathcal{V}^{-11 / 4} \sim 10^{2} \mathrm{GeV}$.

\section{DISCUSSION: INTERPLAY BETWEEN GLOBAL AND LOCAL ISSUES}

In this paper we did not address any of the issues related to the brane construction of the visible sector given that the actual details of the embedding of the Standard Model (or any generalisation thereof like the MSSM or GUT theories) into string theory are completely irrelevant for the study of reheating in the context of closed string inflationary models within the framework of type IIB compactifications.

In fact, type IIB Calabi-Yau flux compactifications are characterised by the fact that physics decouples into local (or brane) and global (or bulk) issues that can be consistently studied separately. Some examples of brane issues that depend only on the local brane construction are finding the right chiral spectrum, gauge group and Yukawa couplings, while issues like moduli stabilisation, supersymmetry breaking, inflation and reheating are purely global.

Regarding the study of the transfer of the inflaton energy density to the Standard Model degrees of freedom, we emphasize that the only thing which has to be considered is the inflaton dynamics after inflation with the nonperturbative (preheating) and perturbative (reheating) particle production. In particular, the study of reheating via the inflaton decay involves only the knowledge of the inflaton mass and couplings to all the degrees of freedom in the theory, and the computation of the overall scaling of these couplings does not depend at all on any local detail of the Standard Model brane construction.

In fact, it is the overall volume of the Calabi-Yau compactification which sets the order of magnitude of all these couplings whose computation requires only the knowledge of the moduli dependence of the gauge kinetic function, the Kähler potential and the superpotential, together with the form of the soft supersymmetry-breaking terms. All these are global or bulk issues. 


\section{CONCLUSIONS}

In this paper we presented a model of closed-string slow-roll inflation embedded in type IIB string compactifications where we have full control over the inflaton dynamics after inflation and the reheating of the visible sector degrees of freedom regardless of the local Standard Model construction.

We tried to bring string inflation closer to 'the real world' describing how to excite the visible sector degrees of freedom in a Calabi-Yau compactification with a robust moduli stabilisation mechanism that allows to check the solution of the $\eta$-problem for inflation and compute the order of magnitude of the moduli mass spectrum and coupling to all particles of the theory.

This paper is mostly based on [8] but it singles out for the first time the best working model of closed string inflation which shows many interesting phenomenological features like the generation of the correct amount of den- sity perturbations, a viable reheating of the visible sector degrees of freedom which can take place substantially before Big-Bang nucleosynthesis, the absence of any cosmological moduli problem, the presence of $\mathrm{TeV}$-scale supersymmetry and the right scale for grand unification theories. We stress that all these achievements can be reached without the need of any fine-tuning.

In this sense, this paper represents a step forward with respect to 8] which was just a general analysis of reheating with the discussion of several models, and the pointing out of some problems that one has to solve to have a viable reheating model.

\section{Acknowledgments}

The research of AM is partly supported by the "UNIVERSENET" (MRTN-CT-2006-035863).
[1] E. Komatsu et al., arXiv:1001.4538 [astro-ph.CO].

[2] A. Mazumdar and J. Rocher, arXiv:1001.0993 [hep-ph].

[3] B. Fields and S. Sarkar, astro-ph/0601514; R. H. Cyburt, Phys. Rev. D 70 (2004) 023505.

[4] R. Allahverdi, K. Enqvist, J. Garcia-Bellido and A. Mazumdar, Phys. Rev. Lett. 97, 191304 (2006); R. Allahverdi, A. Kusenko and A. Mazumdar, JCAP 0707, 018 (2007); R. Allahverdi, K. Enqvist, J. Garcia-Bellido, A. Jokinen and A. Mazumdar, JCAP 0706, 019 (2007).

[5] R. Allahverdi, B. Dutta and A. Mazumdar, Phys. Rev. D 75 (2007) 075018; R. Allahverdi, B. Dutta and A. Mazumdar, Phys. Rev. Lett. 99 (2007) 261301;

[6] J. P. Conlon and F. Quevedo, JHEP 0601 (2006) 146.

[7] M. Cicoli, C. P. Burgess and F. Quevedo, JCAP 0903 (2009) 013; M. Cicoli, Fortsch. Phys. 58 (2010) 115; C. Burgess, M. Cicoli, M. Gomez-Reino, F. Quevedo, G. Tasinato and I. Zavala, JHEP 1008 (2010) 045.

[8] M. Cicoli and A. Mazumdar, JCAP 1009 (2010) 025

[9] J. P. Conlon and F. Quevedo, JCAP 0708 (2007) 019; L. Anguelova, V. Calo and M. Cicoli, JCAP 0910 (2009) 025 .

[10] R. Allahverdi, R. Brandenberger, F. Y. Cyr-Racine and A. Mazumdar, arXiv:1001.2600 [hep-th].

[11] N. Barnaby, C. P. Burgess and J. M. Cline, JCAP 0504
(2005) 007; A. Mazumdar and H. Stoica, Phys. Rev. Lett. 102 (2009) 091601; R. H. Brandenberger, K. Dasgupta and A. C. Davis, Phys. Rev. D 78 (2008) 083502; L. Kofman and P. Yi, Phys. Rev. D 72 (2005) 106001; A. R. Frey, A. Mazumdar and R. C. Myers, Phys. Rev. D 73 (2006) 026003; X. Chen and S. H. Tye, JCAP 0606 (2006) 011; D. R. Green, Phys. Rev. D 76 (2007) 103504.

[12] N. Barnaby, J. R. Bond, Z. Huang and L. Kofman, JCAP 0912 (2009) 021.

[13] S. Kachru, R. Kallosh, A. Linde and S. P. Trivedi, Phys. Rev. D 68 (2003) 046005.

[14] V. Balasubramanian, P. Berglund, J. P. Conlon and F. Quevedo, JHEP 0503 (2005) 007.

[15] M. Cicoli, J. P. Conlon and F. Quevedo, JHEP 0810 (2008) 105.

[16] J. P. Conlon, A. Maharana and F. Quevedo, JHEP 0905 (2009) 109; R. Blumenhagen, J. P. Conlon, S. Krippendorf, S. Moster and F. Quevedo, JHEP 0909 (2009) 007.

[17] M. Berg, M. Haack and B. Kors, JHEP 0511, 030 (2005); M. Berg, M. Haack and E. Pajer, JHEP 0709 (2007) 031; M. Cicoli, J. P. Conlon and F. Quevedo, JHEP 0801 (2008) 052. 